\title{
Didactique de la musique : Apports d'une approche comparatiste
}

Étude des notions de transposition didactique et d'organisation praxéologique pour l'enseignement/apprentissage du piano

\section{Adrien Bourg}

\section{OpenEdition}

\section{Journals}

Édition électronique

URL : http://journals.openedition.org/educationdidactique/264

DOI : 10.4000/educationdidactique.264

ISBN : 978-2-7535-1617-5

ISSN : $2117-4838$

\section{Éditeur}

Presses universitaires de Rennes

\section{Édition imprimée}

Date de publication : 1 juin 2008

Pagination : 69-88

ISBN : 978-2-7535-0712-8

ISSN : 1956-3485

\section{Référence électronique}

Adrien Bourg, «Didactique de la musique : Apports d'une approche comparatiste », Éducation et didactique [En ligne], 2-1 | juin 2008, mis en ligne le 01 juin 2010, consulté le 08 décembre 2020. URL : http://journals.openedition.org/educationdidactique/264; DOI : https://doi.org/10.4000/ educationdidactique.264 


\title{
DIDACTIQUE DE LA MUSIQUE : APPORTS D'UNE APPROCHE COMPARATISTE Étude des notions de transposition didactique et d'organisation praxéologique pour l'enseignement/apprentissage du piano
}

\author{
Adrien Bourg, EDA, Université Paris Descartes
}

\begin{abstract}
Résumé : Cet article est une contribution au développement d'un champ de recherche peu connu, celui de la didactique de la musique, dans le cadre d'une perspective comparatiste. Dans un premier temps, nous rappelons la relative nouveauté de la didactique de la musique au sein des différentes didactiques disciplinaires, mais aussi de l'adoption du point de vue comparatif ; dans un second temps, nous interrogeons le champ de pertinence d'une théorie qui a été développée en didactique des mathématiques - celle de la transposition didactique - pour le domaine de la didactique de la musique. Plus précisément, nous questionnons un objet particulier des savoirs pianistiques - le doigté - en s'appuyant sur la notion d'organisation praxéologique.
\end{abstract}

Mots-clés : didactique de la musique, transposition didactique, organisation praxéologique, piano, doigté.

Adrien Bourg

\section{Introduction}

\section{Didactique de la musique : une intruse dans les didactiques disciplinaires?}

« Les recherches en didactique de la musique existent ». Voici, à l'interchangeabilité d'un mot près (en remplaçant «musique » par «éducation physique et sportive »), un des premiers titres de l'article de Marsenach et Amade-Escot (1993) paru il y a maintenant 15 ans dans la Revue Française de Pédagogie. Cette même année, en 1993, la recherche sur et en éducation musicale, s'est développée dans le paysage universitaire français, sous la forme de la création d'un troisième cycle en Musicologie au sein de l'Université Paris IV-Sorbonne. L'entreprise fut menée sous la direction de J.-P. Mialaret qui a réuni et impulsé toute une génération de jeunes chercheurs (cf. Boudinet \& Fijalkow, 2005). À cette même époque, on pouvait voir figurer dans le Guide bibliographie des didactiques (Desvé, 1993) la didactique de la musique au côté d'autres didactiques disciplinaires ${ }^{1}$. Selon J.P. Mialaret (rédacteur de l'article concernant la didactique de la musique), «l'étude des processus spécifiques d'enseignement-apprentissage de la musique constitue[ait] encore un champ potentiel d'investigation dont l'exploration conduira[ait] véritablement à un renouvellement de la réflexion sur la didactique de la musique » (op. cit., p. 172).
Bien qu'il existe et que se soit développée une littérature scientifique dans le domaine des sciences de l'éducation musicale, aussi bien en ce qui concerne l'étude des conditions générales de l'éducation musicale (selon des perspectives philosophiques, historiques, sociologiques, anthropologiques), que l'étude des conditions de l'acte éducatif en musique (selon des perspectives physiologiques, psychologiques) ${ }^{2}$, on notera que les travaux en didactique de la musique, qui s'intéressent plus spécifiquement aux processus d'acquisition et de transmission des savoirs musicaux sont encore peu nombreux. Pourtant, une réflexion importante sur les apprentissages musicaux avait été initiée dans les années soixante-dix, dans le cadre du courant de l'enseignement programmé. Au sein de l'équipe de Francès, psychologue de la perception musicale, plusieurs recherches se sont ainsi concentrées sur l'étude des processus d'apprentissage de la lecture musicale et sur les conditions de sa transmission (par ex. Francès, 1974; J.-P. Mialaret, 1979). Cette orientation semble ensuite avoir été laissée de côté pour des travaux à consonance plus générale dans lesquels la composante pédagogique prévaut alors sur la composante psychologique.

Pour en revenir aux années quatre-vingt-dix, alors que plusieurs didactiques disciplinaires jouissent déjà d'un avancement certain dans leur domaine, se dessinent de nouveaux questionnements autour de 
la question du comparatisme ${ }^{3}$. À notre connaissance, aucune recherche en musique ne s'est saisie, sinon que tout récemment, de cette « nouvelle» perspective. Aussi, deux voies possibles, bien que non étanches, semblent envisageables afin de poursuivre la construction d'une « didactique de la musique » :

- La première voie, certainement la plus légitime d'un point de vue épistémologique, consisterait à construire une didactique disciplinaire, en partant des questions spécifiques que l'on se pose dans la discipline et a fortiori de la singularité des savoirs qui la composent, et de construire des instruments méthodologiques et théoriques adaptés aux problématiques et objets que l'on se donne et que l'on souhaite observer. La didactique des mathématiques, non sans l'influence des outils et concepts développés dans d'autres domaines des sciences humaines (notamment la psychologie, l'épistémologie, la sociologie, l'anthropologie), a ainsi développé ses propres problématiques, méthodologies et théories.

- La deuxième voie consisterait à introduire, pour une discipline donnée, des concepts nés dans d'autres didactiques disciplinaires. Cette seconde démarche n'évacue nullement la possibilité, envisagée ici comme une nécessité, d'adapter les outils tout autant que les concepts à la spécificité de la discipline étudiée. Dans un autre temps, cette démarche peut d'ailleurs être complétée par un retour théorique au champ de la didactique dans lequel le concept a émergé, soit amener des avancés et contribuer à l'évolution en retour des concepts initiaux.

Cette seconde perspective, dans laquelle nous nous situons, ne constitue pas une voie concurrente à la première, encore moins une voie alternative; elle doit se concevoir comme un accompagnement épistémologique de la discipline ${ }^{4}$, et nous semble un accélérateur nécessaire à la construction d'une plus grande unité de la recherche dans notre domaine. Elle peut, nous semble-t-il, contribuer ainsi à la fois à ouvrir des perspectives de recherche pour les futurs travaux en didactique de la musique, en empruntant et interrogeant de manière critique certains concepts extérieurs à la discipline, tout comme elle peut participer à enrichir les travaux dans le champ de la didactique comparée. Certains pourraient rétorquer, selon une autre vision, que l'on pourrait également concevoir l'approche comparatiste comme une confronta- tion a posteriori des systèmes théoriques développés par les différentes didactiques des disciplines (qui se sont constituées, dans une certaine mesure, indépendamment les unes des autres). Cependant, le caractère nouveau et peut-être encore trop disparate de la recherche en didactique de la musique, ne nous permet pas, selon nous, de développer à l'heure actuelle une comparaison (du moins systématique) sur la base de ce principe.

Transposition didactique: le cas de
l'enseignement pianistique

\section{Un positionnement théorique}

Nous interrogeons dans cette communication le champ de pertinence d'une théorie qui a été développée en didactique des mathématiques, la théorie de la transposition didactique, pour le domaine de la didactique de la musique. Malgré les nombreuses critiques systématiquement émises notamment sur la question du(es) « savoir(s) savant(s)» (Raisky \& Caillot, 1996), mais aussi grâce au nouvel appui que représente la notion d'organisation praxéologique pour la description des savoirs (telle qu'elle est définie dans le cadre de la théorie anthropologique du didactique), nous reprenons, après d'autres didactiques, la démarche qui consiste à interroger cette théorie dans un autre champ disciplinaire, autre que celui d'origine. S'inscrire dans ce type de questionnement, ce n'est évidemment pas nier l'importance du champ disciplinaire spécifique qui a permis l'émergence de cette théorie; c'est penser les limites du domaine de validité d'une théorie, l'autonomie des théories audelà de leur contexte d'émergence.

Nous concevons avant tout la théorie de la transposition didactique, comme un outil heuristique, aidant à la modélisation de certains phénomènes didactiques. Aussi, nous avons souhaité dépasser certains clivages constatés dans les différentes didactiques disciplinaires, entre deux théories apparemment concurrentes : celle de la Transposition didactique (développée en didactique des mathématiques par Chevallard, 1985, suite aux travaux du sociologue Verret, 1975) et celle des Pratiques sociales de référence (développée en didactique des sciences expérimentales et techniques par Martinand, 1986) ${ }^{5}$. Même 
si ces deux théories s'inscrivent dans des démarches et finalités différentes, et qu'elles ont chacune un champ de questionnement qui leur est propre (Bourg, 2006a), elles ont une fonction commune: elles permettent d'interroger la distance entre les savoirs extérieurs au système scolaire et le système scolaire dans une perspective descriptive et épistémologique. Il s'agit d'observer comment vit et fonctionne un savoir (ou plus globalement des pratiques) dans différentes institutions, de montrer les contraintes qui pèsent sur le fonctionnement du système d'enseignement, et de poser le problème de la légitimation d'un contenu d'enseignement. Nous retenons ainsi l'idée, non étrangère à la théorie anthropologique du didactique, que les savoirs peuvent s'observer à travers l'étude de différentes institutions, et que la manière dont ils fonctionnent peut faire l'objet de comparaisons.

Létude présentée ici prend pour cadre l'enseignement pianistique dans les conservatoires de musique. Elle s'appuie sur deux postulats : l'un considère que s'il y a pour une discipline donnée un enseignement, c'est qu'il existe bien des savoirs (dans le sens où nous le développerons en nous appuyant sur la notion d'organisation praxéologique), l'autre affirme que tout savoir subit des transformations dans le but d'être enseigné. On notera que ces postulats constituent une réaction vive contre, d'une part les idéologies encore persistantes sur le don en musique (qui ont certainement constitué un obstacle au développement de la recherche en ce domaine), et d'autre part envers certains discours sur la musique qui renvoient systématiquement celle-ci à son aspect indicible.

Le premier postulat constitue une réaction vive aux propos de Verret (1975) qui soutient que « tout savoir n'est pas scolarisable, en particulier les savoirs empiriques » (p. 147). Nous pensons que s'il existe un enseignement, dans notre cas l'enseignement d'un instrument de musique, c'est qu'il existe bien des "savoirs", mêmes s'ils sont insérés dans des pratiques et dans des jeux de langages particuliers. Sans reprendre les débats qui ont lieu autour de la question de la prise en charge de pratiques ou de savoirs dans le cadre de la transposition didactique, soulignons que notre position consiste à penser que la transposition ne peut prendre directement des pratiques comme "référence ", on s'appuie forcément sur une représentation de ces pratiques, sur un modèle de la pratique, sur des pratiques objectivées. Les pratiques en tant que telles, les pratiques réelles, ne peuvent donner lieu directement à un enseignement-apprentissage, il s'agirait alors, comme le note Johsua (1996), d'un apprentissage de type mimétique ou initiatique, même si ces dimensions ne sont pas à négliger dans l'enseignement-apprentissage d'un instrument de musique. Le postulat avancé repose avant tout sur le fait que la pratique pianistique s'enseigne: d'une part, il existe un enseignement institutionnalisé, notamment dans les conservatoires de musique, avec une division par cycle et par niveau des enseignements, d'autre part, il existe des méthodes de piano qui proposent une " mise en texte » de savoirs délimités et ordonnés dans le temps. Néanmoins, on soulignera que contrairement au milieu scolaire, où les savoirs musicaux à enseigner transparaissent par l'intermédiaire des programmes et des textes officiels (on enseigne d'ailleurs l'« éducation musicale », non la «musique »), dans les conservatoires de musique il n'existe pas de "programmes ", tout juste quelques directives pédagogiques d'un caractère assez général ${ }^{6}$. Les professeurs dépourvus de tout « savoir à enseigner » explicite, doivent alors composer leur enseignement en fonction de leur expérience et des contraintes institutionnelles. Autrefois, une instance légitime, le CNSM (Conservatoire National Supérieur de Musique), était chargée de «dire le vrai » dans l'enseignement de la musique en France, notamment par la diffusion des méthodes réalisées par des professeurs de cette institution (cf. Hondré, 1995). Soulignons enfin que ce postulat repose avant tout sur l'idée que les savoirs pianistiques, même s'ils ne jouissent pas tous de la même visibilité, peuvent cependant être formalisés en partie par le chercheur, suivant une objectivation des éléments qui composent la pratique pianistique sur la base du repère d'un ensemble d'invariants.

Le deuxième postulat, qui affirme que tout savoir subit des transformations dès lors qu'il est assujetti à un enseignement, renvoie directement à la théorie de Chevallard (1985). Si nous considérons que la pratique pianistique peut être appréhendée en termes de savoirs (plus précisément en termes de praxéologies), dès lors elle est soumise aux mêmes contraintes que les savoirs que l'on pourrait qualifier de plus académiques. Ces contraintes agissent à tous les niveaux d'enseignement et d'apprentissage, dès lors qu'il y a une intention didactique. Aussi, nous sommes en désaccord avec les 
propos de Schillinger (1995) lorsque celui-ci affirme qu' « il n'y a pas [dans les CNSM] à ce niveau d'étude, de transposition didactique, (...), les savoirs enseignés sont les savoirs savants » (p. 223). S'il est vrai en effet que les enseignants de ces conservatoires représentent l'élite de référence de la pratique musicale en France, tout comme les élèves, qui sont susceptibles de devenir les futurs concertistes de demain, il n'en est pas moins vrai que toute forme d'enseignement, quel que soit le niveau, voit son savoir transposé (ne serait-ce qu'en prenant pour critères le fait qu'il existe dans ces structures institutionnelles un cursus qui se décompose en niveaux définis, et que le conservatoire eut très tôt la volonté d'organiser la progression des apprentissages en proposant des méthodes instrumentales).

\section{Un objet : le doigté}

Dans le cadre de la pratique pianistique et de son enseignement, nous analysons les mécanismes transpositifs d'un objet précis des savoirs pianistiques - le doigté - relativement à un morceau ${ }^{7}$ extrait d'un recueil à usage didactique.

Le doigté est un élément du savoir pianistique que l'on peut qualifier d'incontournable, puisqu'il est la condition sine qua non pour jouer d'un instrument à clavier. Si les doigtés ${ }^{8}$ peuvent figurer sur la partition (on les indique généralement au-dessus des notes), souvent les compositeurs ne les indiquent que partiellement voire pas du tout ${ }^{9}$. Les partitions proposées à l'étude ne comportent ainsi aucun doigté stipulé par le compositeur. Cependant les éditeurs qui révisent les partitions (en s'entourant de « doigteurs professionnels ») peuvent doigter la totalité ou une partie des morceaux ${ }^{10}$, à partir desquels les pianistes débutants ou confirmés travailleront. Lorsqu'il n'y a pas de doigtés indiqués sur la partition, le pianiste se retrouve alors dans une situation où il doit faire des choix sur les doigtés à employer.

En effet, contrairement à d'autres instruments où un doigté correspond le plus souvent à une note, au piano une même note peut être exécutée avec différents doigtés. Ainsi, une même configuration mélodico-rythmique pourra être exécutée avec des séries de doigtés différentes. Prenons pour exemple, la tâche consistant à exécuter le groupe de trois notes conjointes suivant : fa\#-sol-la. Une énuméra- tion théorique des combinaisons de doigtés possibles (pour une main) nous conduit au calcul suivant : $5 \mathrm{x}$ $4^{\mathrm{n}-1}(\mathrm{n}=$ nombre de notes $)$, soit pour trois notes, 80 possibilités $(121,123,124,125,131,132,134, \ldots .345)$. Cependant tous les doigtés ne se valent pas, on pourra recueillir dans les discours de pianistes les expressions suivantes : ils opposent le vrai doigté au faux, le bon au mauvais, le commode à l'incommode ou au dangereux, le simple au complexe, l'académique à l'orignal. Une des variables importante dans l'exécution d'un doigté est celle qui se rapporte aux contraintes physiologiques et motrices, c'est ce que prend en partie en charge le modèle ergonomique du doigté proposé par Parncutt, Sloboda, Clarke, Raekallio, et Desain (1997). Ce modèle repose sur les limitations anatomiques de la main (notamment l'empan, ou écart maximum réalisable entre des paires de doigts; cf. Wagner, 1988). Pour notre tâche, le modèle en évacuant les séries de doigtés impraticables, fait tomber les combinaisons de doigtés de 80 à $28(121,123,124,125,131,134,135,141,145$, 212, 213, 214, 215, 231, 234, 235, 241, 245, 312, 313, 314, 315, 341, 345, 412, 413, 414, 415; cf. figure 1). Dans un second temps, toujours dans ce modèle, les successions de doigtés possibles sont hiérarchisées en fonction de leur niveau de difficulté, estimé en fonction d'un système de 12 règles. Chaque règle représente une source spécifique de difficulté ergonomique (relativement aux variations de la force et de l'agilité des doigts, à l'exécution de certains doigts mise en rapport avec des aspects liés à la topographie du clavier, etc.) ${ }^{11}$.

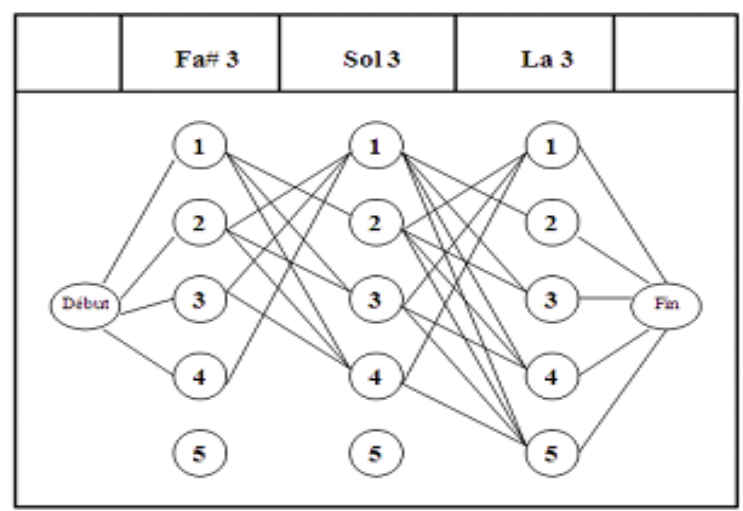

Figure 1 : Énumération des combinaisons de doigtés praticables d'après Parncutt et al. (1997) (les chiffres correspondent aux doigtés possibles pour chacune des notes; le schéma se lit de gauche à droite de "début " à "fin » en suivant les trajets) 
Ainsi, si de manière restreinte le "doigté » désigne les doigts qui doivent actionner les touches du clavier, il réfère également à un processus complexe, qu'est l'action de doigter. La situation est alors assez analogue à l'activité d'un élève qui résout un problème. On pourrait considérer la recherche de doigtés comme répondant à une sorte de situationproblème, dont la partition constituerait l'énoncé, et que l'on doit résoudre sans connaître de manière explicite les composantes (de cet énoncé) et sans qu'il existe de solutions uniques et définitives (domaine de l'interprétation). Cependant, pour une tâche donnée il existe des constantes dans le choix des doigtés utilisés (Bourg, 2006b), qui semblent être déterminées par une série de contraintes anatomiques et motrices, mais aussi par des choix plus individuels, et par l'héritage d'usages techniques et d'interprétations aujourd'hui en partie stabilisés et partagés par un ensemble de pianistes.

\section{L'étude d'institutions}

Dans le cadre de la pratique instrumentale (en occident) ${ }^{12}$, on distinguera trois grandes institutions qui sont impliquées dans le processus de didactisation des objets de savoirs (cf. figure 2) :

- celle des experts : dans notre étude il s'agit des pianistes-concertistes, leurs pratiques correspondent aux pratiques musicales de référence de la pratique pianistique, telle que nous l'étudions à travers les structures institutionnelles particulières que sont les conservatoires municipaux de musique. De ces pratiques il est possible de déterminer des «savoirs experts » (expression de Johsua, 1998) de référence suivant une démarche de formalisation qui consiste à repérer des invariants, à identifier, comme le proposent Rogalski et Samurçay (1994), « des catégories d'objets et de traitements communes à des pratiques efficaces, qui sont quant à elles spécifiques de situations contextualisées et personnalisées » (p. 43);

- celle des éditeurs de musique: au sein de leur activité les éditeurs s'entourent d'un ensemble d'acteurs, pianistes, compositeurs, musicologues ou pédagogues, qui peuvent revêtir la fonction de « doigteur professionnel». C'est dans cette institution que s'opère un premier processus de transposition didactique, visible à travers les nombreuses transformations que subit le manuscrit autographe du compositeur jusqu'à ce que la partition soit éditée. $\mathrm{Au}$ cours de ce processus les éditeurs font des choix vis-à-vis des objets de savoirs qu'ils adaptent en fonction du public visé (cf. Bourg, 2007);

- celle des cours de piano : notre étude s'intéresse à l'enseignement qui a lieu dans les conservatoires municipaux de musique ${ }^{13}$. On pourra distinguer dans l'institution « cours de piano » deux topos, celui du professeur, et celui de l'élève. Il s'agit d'observer ici les savoirs pianistiques réellement enseignés. Les professeurs de piano sélectionnent à leur tour (après le travail des éditeurs) le savoir qu'ils souhaitent enseigner et l'adaptent en fonction de leurs élèves. On notera par ailleurs que les pratiques des éditeurs et des professeurs ont une incidence sur la manière dont les élèves apprennent et mobilisent des procédures particulières (Bourg, 2006b).

À partir de ces institutions il est alors possible d'analyser de manière comparative la manière dont "vivent » les objets de savoirs. La figure ci-dessous (figure 2) présente sous une forme schématisée les différentes institutions que l'on peut étudier dans leurs rapports réciproques relativement à l'objet " doigté » (objet considéré dans le cadre de l'étude d'une partition en particulier). 


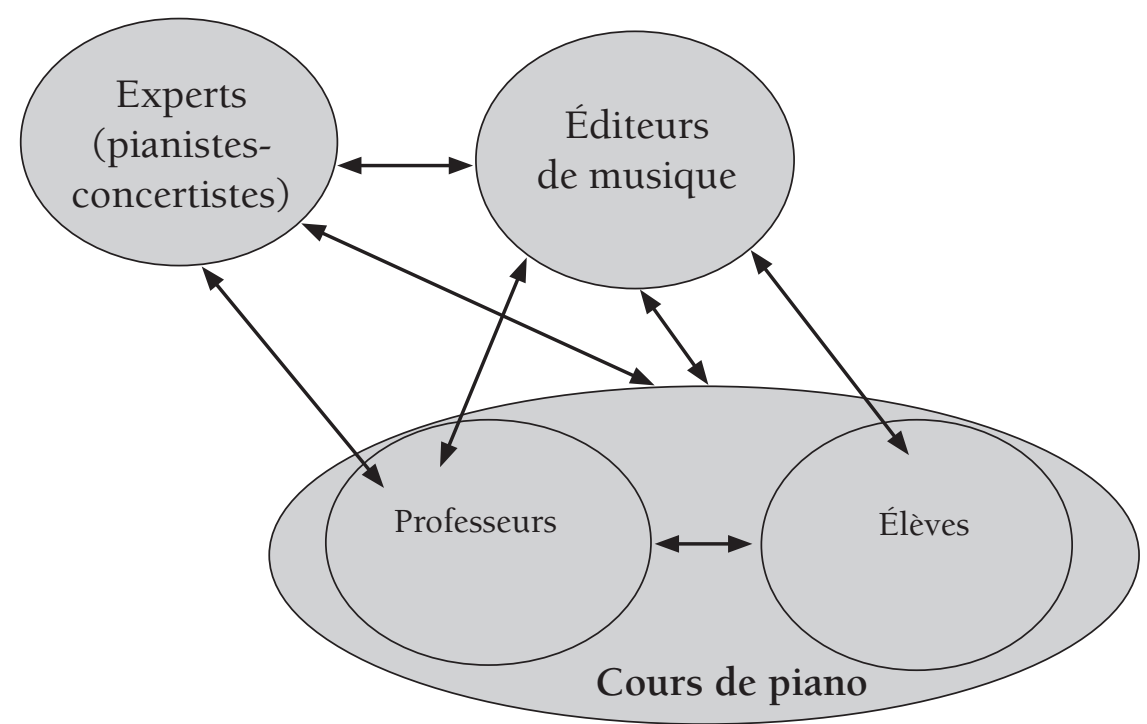

Figure 2 : présentation schématique des institutions impliquées, pour la pratique instrumentale, dans l'étude de phénomènes de transposition didactique

\section{L'étude des savoirs pianistiques}

\section{La notion d'organisation praxéologique}

Pour décrire et analyser les pratiques de doigtés dans les différentes institutions précitées, nous nous appuyons sur la notion d'organisation praxéologique que développe Chevallard dans le cadre de la théorie anthropologique du didactique (1996, 1999a, 1999b).

Pour cet auteur, la praxéologie désigne à la fois la praxis, le savoir-faire, et le logos, le savoir. Dans cette perspective, la didactique a pour objet « les praxéologies et les conditions et contraintes de leur diffusion sociale » (Chevallard, 2007, p. 18). Plus précisément, Chevallard propose d'étudier les pratiques, à partir de la notion d'organisation praxéologique, définie par le quadruplet : tâche - technique - technologie - théorie. L'auteur distingue des tâches routinières, des tâches problématiques, ces dernières comportant des difficultés susceptibles d'empêcher un individu d'accomplir certains types de tâches. (Une tâche problématique peut d'ailleurs devenir routinière). La tâche ne se réalise aussi que par la mise en œuvre d'une technique déterminée, " une manière de faire ». Celle-ci est définie comme « un ensemble réglé de gestes que l'on accomplit dans un certain dispositif » (1996, p. 87). Le « discours» qui rend compréhensible et qui justifie une technique est appelé technologie. Dans les organisations praxéologiques il existe enfin un troisième niveau qui correspond à une sorte de technologie de la technologie, que Chevallard nomme la théorie. Il s'agit d'un "discours» qui a pour objectif de justifier et d'expliquer la technologie. Lauteur distingue ainsi deux blocs, un premier bloc pratico-technique qui correspond au savoirfaire, et un deuxième bloc technologico-théorique, que l'on pourrait identifier à un savoir (cf. figure 3 ). Lauteur précise qu'« on ne parlera donc en général de savoir ou de savoir-faire que par métonymie, savoir et savoir-faire étant normalement pris ensemble dans l'unité d'une praxéologie » (1997, p. 4). 


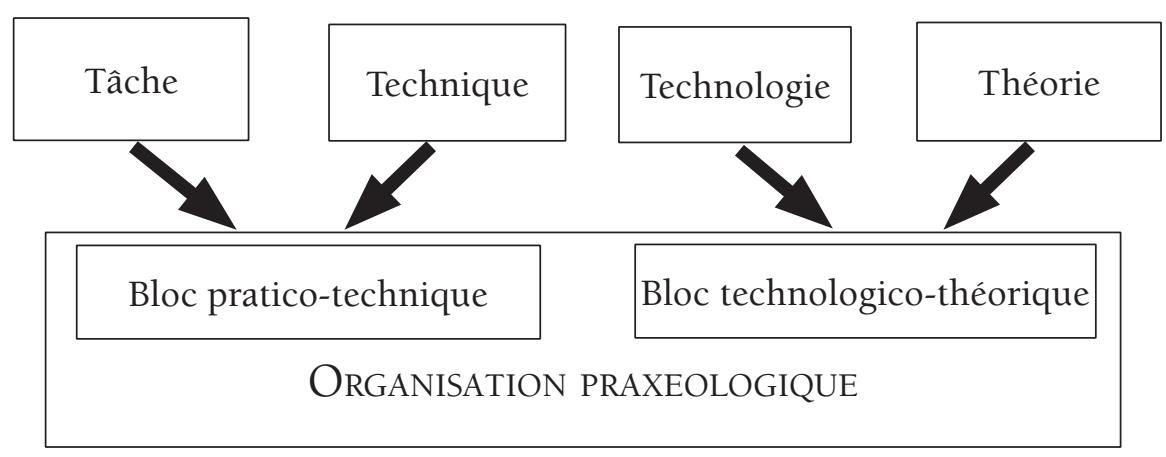

Figure 3 : la notion d'organisation praxéologique (d'après Chevallard, 1996)

\section{Des praxéologies pianistiques}

La notion d'organisation praxéologique est séduisante à plusieurs égards: d'une part elle permet de mettre à distance la vision dichotomique commune qui considère certaines activités comme manuelles, alors que d'autres seraient intellectuelles, d'autre part elle propose un cadre théorique qui permet de penser d'un point de vue épistémologique et didactique les objets de savoirs. La réflexion qui suit, tente de montrer l'intérêt de cette notion pour la description des pratiques pianistiques, même si nous formulerons certaines critiques à son égard. Nous emprunterons des exemples issus d'une analyse historique systématique de traités et méthodes de piano (Bourg, 2006b) provenant d'un corpus s'échelonnant $\mathrm{du} \mathrm{xVI}^{\mathrm{e}}$ siècle (époque où apparaissent les premiers traités pour instruments à clavier) à aujourd'hui.

La figure ci-jointe (figure 4) montre, pour une même tâche, les différents doigtés (parmi parfois plusieurs propositions) recommandés par des auteurs de traités. La tâche consiste en l'exécution d'une gamme ascendante de do majeur à la main droite, effectuée sur l'espace d'une octave.

\begin{tabular}{|c|c|c|c|c|c|c|c|c|}
\hline C.P.E Bach (1753) & 1 & 2 & 3 & 1 & 2 & 3 & 4 & 5 \\
\hline & & & & $a$ & 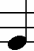 & 0 & 0 & $e$ \\
\hline Marpurg (1750) & 1 & 2 & 3 & 4 & 1 & 2 & 3 & 4 \\
\hline Santa Maria (1565) & 3 & 4 & 3 & 4 & 3 & 4 & 3 & 4 \\
\hline Venegas (1557) & 1 & 2 & 3 & 4 & 3 & 4 & 3 & 4 \\
\hline Buchner (1520) & 2 & 3 & 2 & 3 & 2 & 3 & 4 & \\
\hline
\end{tabular}

Figure 4 : exemples de l'évolution des doigtés de la gamme de do majeur pour la main droite 
Cette tâche peut être exécutée en utilisant des configurations de doigtés différentes : en faisant se succéder les $2 \mathrm{e}$ et $3 \mathrm{e}$ doigts (Buchner), les $3 e$ et $4 \mathrm{e}$ doigts (Santa Maria, Venegas), ou encore en utilisant le pouce (Marpurg, C.P.E. Bach). Ces doigtés sollicitent des techniques pianistiques spécifiques mettant en jeu soit le chevauchement des doigts, soit le passage du pouce.

Ces techniques sont marquées historiquement, géographiquement et esthétiquement. La présentation chronologique des différents doigtés de la figure 4, montre une évolution dans la « manière » de doigter (actuellement on utilise conventionnellement le doigté qu'utilise C.P.E. Bach dans cet exemple). Cette évolution n'exclut cependant pas la possibilité d'utiliser à une époque donnée des doigtés employés à des époques antérieures. Les auteurs proposent souvent plusieurs doigtés, qu'ils aiment à caractériser au $\mathrm{XVIII}^{\mathrm{e}}$ siècle en faisant référence aux transformations des usages. Ainsi peut-on lire les expressions suivantes dans quelques traités: "ancien usage", «nouveau usage ", "façon ancienne », "façon nouvelle », «à la moderne » (Couperin, 1717), «à la mode » (C.P.E. Bach, 1753), « principe anciennement établi », « doigter (sic.) moderne » (Viguerie, 1796), « doigtés d'aujourd'hui » (Pleyel et Dussek, 1797). Certains auteurs, comme Saint Lambert (1702) dévoilent même l'état de transformation d'un usage et du problème de sa stabilité : «(...) mais j'ajoute icy que cet usage ne me paroit pas encore bien établi et que le pouce et le second doigt conviendroient mieux à ces sortes de passages (...)» (p. 65). La transformation d'un ancien usage à un nouveau est souvent présentée comme une nécessité, à laquelle s'ajoute la difficulté de l'abandon des « diverses sortes d'habitudes " (Nonot, 1797, p. 2). Duphly (1769) ou Viguerie (1796) utilisent par exemple l'expression « s'accoutumer à passer le pouce », concernant l'utilisation du premier doigt au sein des mouvements de gammes.

Comme le dénote cette dernière expression, l'évolution des techniques employées relativement à ce type de tâche est marquée par la participation du pouce dans le jeu pianistique qui n'intervient de manière plus systématique qu'à partir du milieu du $\mathrm{XVIII}^{\mathrm{e}}$ siècle. (On trouvera cependant quelques exceptions, comme l'utilisation de configurations de type 1234-1234 déjà présentes chez Bermudo, 1555). Cette évolution de la participation du pouce dans le jeu pianistique est soulignée par certains auteurs comme C.P.E. Bach (1753) qui rapporte ces quelques mots de son père : "Mon défunt père me racontait qu'il avait pu entendre de grands musiciens dans sa jeunesse qui ne se servaient de leur pouce que lorsqu'une grande extension l'exigeait. Il vivait en un temps où, petit à petit, un changement très particulier s'effectuait dans le goût musical (...) Ainsi est-il sorti (le pouce) de l'inutilité pour devenir un des doigts importants » (\$7). Fétis (1840) nous rapporte même que cet emploi particulier du pouce a été longtemps connu en Allemagne sous le nom de « Doigté de [J.S.] Bach » (Introduction, p. 2). L'usage plus systématique de ce doigt au cours du XvIII ${ }^{\mathrm{e}}$ siècle sera en fait revendiqué dans les méthodes par des conseils et des exercices appropriés, pour constituer au XIX ${ }^{\mathrm{e}}$ siècle une clé de voûte de la technique pianistique. Ainsi, Pleyel et Dussek (1797) déclareront que « (...) l'art du doigté consiste principalement à bien entendre le vrai maniement du pouce (...)» (p. 19), ou encore Hummel (1828) invoquera que «le doigt le plus important est le pouce; c'est le point d'appui autour duquel les autres doivent agir (...)» (p. 105).

Lemploi de l'une ou de l'autre de ces techniques (chevauchement des doigts ou passage du pouce) a également un impact sur le résultat sonore de la production musicale. La distinction au $\mathrm{XvI}^{\mathrm{e}}$ siècle entre « bons doigts » et « mauvais doigts » insiste sur une hiérarchisation des doigts mise en rapport avec les temps métriquement forts ou faibles. Diruta (1593), rédigeant les leçons de Merulo, distingue ainsi les « bons doigts », que sont l'index et l'annulaire, des " mauvais doigts ", pouce, majeur et auriculaire, qu'il faut réserver aux notes faibles (cf. figure 5, ex.1). Le doigté alors utilisé induit une façon particulière de phraser «deux en deux » les notes. Cette manière de faire est d'ailleurs radicalement différente de ce qui se faisait chez les contemporains de Diruta, qui utilisaient pourtant le même principe de chevauchement des doigts (cf. figure 5, ex.2). Cette technique amène ainsi à l'exécution un phrasé particulier, avec une exécution des croches le plus souvent inégale ${ }^{14}$, alors que la technique du passage de pouce tend au contraire à gommer ces inégalités, et considérer chacune des notes (et des doigts) comme étant égale de l'autre. Ainsi en témoignent Adam et Lachnitz (1798) : « En passant le pouce par-dessous les doigts, ou les doigts par-dessus le pouce, on doit lier les tons 
de manière qu'on n'entende pas ce changement de doigts; il faut qu'il n'y ait aucune interruption dans le trait ou dans le chant, et que tous les tons soient égaux en force » (V). On sait néanmoins, que cette optique d'« égalité tant pour la durée du tems (sic.) que pour la force du son» (Clementi, 1801, p. 15), reste un idéal qui ne sera jamais atteint. En effet, certaines recherches ont mis en évidence, en fonc- tion des doigtés utilisés, et particulièrement lors du passage du pouce, des irrégularités dans les intervalles de temps entre les notes et dans les nuances. Par exemple, l'utilisation du pouce est marquée par une augmentation de la vitesse d'enfoncement des touches lorsqu'on monte la gamme, alors que c'est l'inverse lorsqu'on la descend (Mac Kenzie \& Dwayne VanEerd, 1990; voir aussi Robine, 2006).

Ex.1: à la manière de Diruta

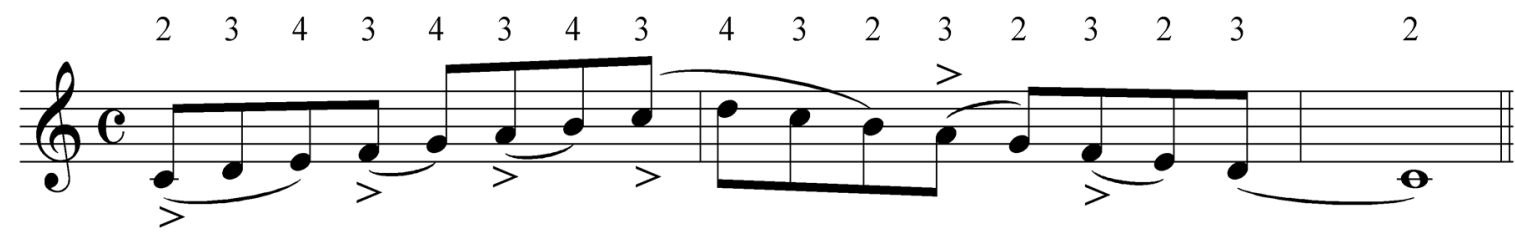

Ex.2: à la manière des Virginalistes anglais

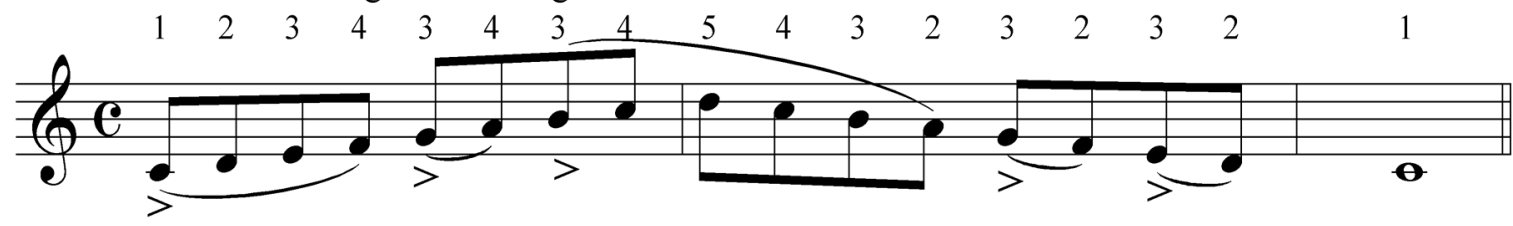

Figure 5: différentes manières de phraser la gamme pour la main droite à la fin du XVI siècle

Le fait que différents doigtés induisent la mobilisation de techniques différentes, n'exclut pas qu'on pourra également repérer pour une même configuration de doigtés des techniques différentes. Le passage du pouce, peut ainsi se faire en passant le pouce au-dessous des autres doigts, ou encore audessus. Par exemple Corette (1749) propose pour monter « l'octave ", l'utilisation du passage du pouce après le quatrième doigt, en faisant passer le pouce en montant « par-dessus » le $4^{e}$ doigt (E). Cette technique constitue en fait l'héritage de l'ancienne façon préconisée encore par cet auteur (technique utilisant le chevauchement des doigts). Par la suite d'autres auteurs, comme Pleyel et Dussek (1797) décriront de manière précise la mouvance du pouce «pardessous » les autres doigts en nous demandant d'être attentif lors de ce mouvement à ne pas « déplacer la position propre de la main » (p. 18).

On pourrait encore raffiner les catégories relatives aux aspects moteurs qui sont liés à ces techniques. Par exemple, concernant le passage du pouce au-dessous des autres doigts, les auteurs des traités décrivent différentes manières d'incliner la main. Contrairement à certaines écoles qui préconisent de jouer la main perpendiculairement au piano, Baduraskoda (1974) indique par exemple que « la meilleure position de la main pour jouer les gammes est une position 'en diagonale', autrement dit avec les bras faisant un angle aigu ou un angle obtus par rapport au clavier ». Cette inclinaison manuelle faciliterait ainsi le passage du pouce. Nous sommes ici arrivés au niveau du discours qui décrit la technique mais aussi qui la justifie, notamment avec certains travaux, comme ceux d'Ortmann (1929) qui montre par exemple dans des expériences, relativement précises pour l'époque, que du point de vue physiologique la supination favorise l'aisance du jeu et de la force.

On soulignera néanmoins que peu de traités (c'est le cas aussi des pianistes) émettent des discours afin de justifier l'emploi des techniques. Les quelques rares justifications que l'on trouve peuvent porter sur des considérations musicales, esthétiques, ou encore 
motrices. On pourra ainsi évoquer les différences de force entre les doigts pour justifier d'une technique, et plus spécifiquement s'appuyer sur la « science du mouvement », comme le décrit Breithaupt (1921), qui distingue notamment les technologues modernes du piano des anciens, par l'introduction des principes de pesanteur, d'impulsion et de rotation. Les considérations technologiques ont certainement d'ailleurs participé à changer le regard des pianistes sur certaines techniques. Par exemple Tetzel (1927) montre qu'une fois la corde frappée par le marteau, rien ne peut modifier le son, et par conséquent que « toute pression, tout massage ou balancement sur la touche restent désormais inutiles» (p. 30). On voit ici que la technique est susceptible d'être modifiée par un discours technologique, qui est sous-tendu par des théories sur les propriétés mécaniques et acoustiques de l'instrument. Si Chevallard rend compte en mathématiques d'un troisième niveau - la théorie - qui est un « discours » qui a pour objectif de justifier la technologie, il faut soulever la difficulté de remonter en musique à ce niveau que constitue la théorie. Niveau, qui cependant même en mathématiques, comme le note Chevallard (1996), n'apparaît souvent qu'à l'état de trace. L'exception en musique consisterait à considérer des technologies qui réfèrent principalement à des aspects physiologiques et moteurs de la technique, technologies qui pourraient alors se justifier par des théories motrices. Il s'agirait alors de théories importées, qui n'ont pas le même statut que les théories auxquelles fait appel Chevallard.

\section{Une analyse de praxéologies pianistiques dans différentes institutions}

Nous proposons d'analyser, à travers l'étude d'un fragment musical, la manière dont fonctionne le doigté dans les différentes institutions que nous avions préalablement définies. Il s'agit d'étudier à partir d'une tâche donnée, les différents doigtés qu'utilisent les pianistes-concertistes, d'observer les doigtés que proposent les éditeurs mais aussi les professeurs de piano, ainsi que d'analyser les discours qui viennent justifier leur emploi. Nous menons nos analyses à partir de plusieurs données : du côté des experts ${ }^{15}$, nous avons enregistré les doigtés de six pianistes lors d'une interprétation du morceau (nous avons également mené des entretiens et proposé des tâches expérimentales); du côté des éditeurs, l'analyse des doigtés a reposé sur un corpus composé de 38 éditions différentes du même morceau; du côté des professeurs de piano ${ }^{16}$, nous avons interrogé six enseignants relativement aux extraits présentés en les questionnant sur ce qu'ils proposent comme doigté à leurs élèves et en leur demandant de se situer par rapport aux choix de certains éditeurs.

Le fragment musical soumis à l'analyse provient d'un morceau, la Musette en ré majeur (BWV Anhang 126), extrait du Petit livre d'Anna Magdalena Bach (1725). Ce morceau est avant tout destiné à de jeunes pianistes (on en fait communément son étude à partir de 2 ans de piano environ), même s'il se voit également être interprété par des concertistes dans le cadre de l'exécution de l'ensemble des pièces du cahier. Nous sommes attentifs dans l'extrait choisi, qui ne comporte originellement aucun doigté, aux doigtés employés pour exécuter le fa\# du premier temps de la troisième mesure qui se joue à la main droite (voir la note entourée, figure 6). Les propositions de doigtés gravitent autour de deux suggestions : soit l'emploi d'un $2^{\mathrm{e}}$ doigt, soit celui d'un $3^{\mathrm{e}}$ doigt. Nous analyserons plus particulièrement les deux séries de doigtés les plus utilisées par l'ensemble des acteurs : 34543253 et 234321421 . 


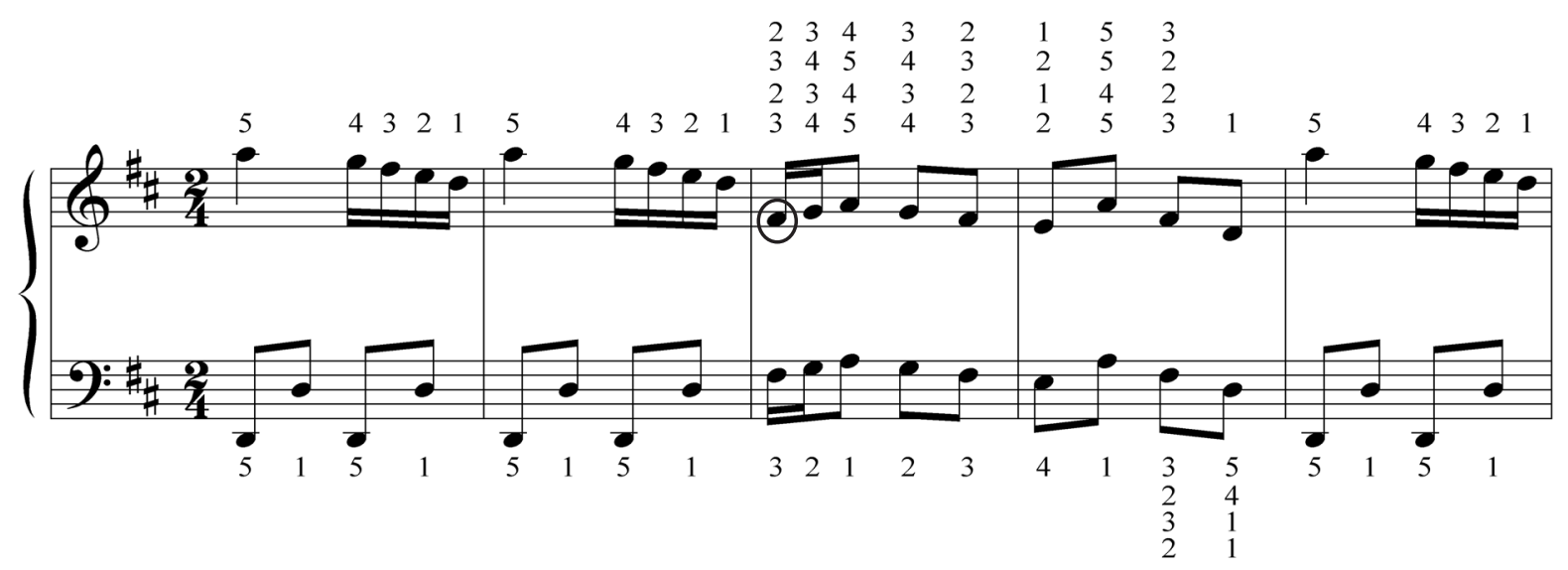

Figure 6 : doigtés proposés par les éditeurs sur les 5 premières mesures de la Musette (présentation par fréquence d'utilisation: du doigté le plus utilisé, près des notes, au doigté le moins utilisé, excentré des notes)

Dans cet extrait, la moitié des pianistes joue le fa\# avec un deuxième doigt, alors que les éditeurs proposent en majorité (85\%) l'exécution de ce passage avec un $3^{\text {e }}$ doigt, tout comme les professeurs de piano. Le doigté proposé diffère ainsi en fonction des institutions dans lesquelles on se situe. Néanmoins, les justifications apportées sur le doigté employé varient peu en fonction des institutions, la différence revient au choix qui sera finalement pris pour l'exécution. Ainsi, l'emploi du deuxième doigt est justifié par la prise en compte des doigts les plus agiles afin d'obtenir une meilleur articulation du premier groupe de notes (fa\#-sol-la-sol-fa\#); l'utilisation du troisième doigt, davantage prescrite par les éditeurs et les professeurs, est justifiée par la prise en compte principalement de deux aspects : le premier renvoie à la notion de position, le deuxième à des aspects liés à la coordination temporelle des gestes bimanuels :

- La notion de position réfère aux notes que l'on peut jouer à partir d'un emplacement fixe des doigts sur le clavier: les mesures 3 et 4 peuvent ainsi être jouées d'une embrassade (sans extension des doigts ou de déplacement latéral du bras), simplement en posant chacun des doigts de la main sur une note de la position de ré (on jouera le ré avec le pouce, le mi avec l'index, le fa\# avec le majeur, etc.). On soulignera, sur un plan historique, que la notion de position a conduit à un renouvellement de la manière de doigter. Elle permet notamment à Pleyel et Dussek (1797) de justifier l'emploi de certaines techniques comme le passage du pouce sous les autres doigts dans les passages qui comportent plusieurs notes de suite, tout comme elle évacue en même temps l'emploi de certaines techniques, comme le croisement des doigts, considéré alors comme non « naturel». Ces auteurs fondent en fait leur système de doigté à l'image de la première règle qu'ils énoncent « $1^{\circ}$ ne changer la position de la main que le moins possible $»$ (p. 39).

- Le deuxième aspect est lié au problème de coordination entre les deux mains. Soulignons avant toute chose que l'exécution de l'extrait pour la main gauche impose un seul doigté envisageable, avec l'utilisation d'un $3^{\text {e }}$ doigt sur le fa\#; comme le préconisait C.P.E. Bach (1753) le doigt long (le majeur) repose ici «naturellement » sur la touche noire, selon une adéquation idéale de la morphologie des doigts avec la topographie du clavier. En proposant un troisième doigt pour la main droite, et donc pour les deux mains, dans le cadre de l'exécution de mouvements parallèles à l'octave, les éditeurs et professeurs proposent une configuration de doigtés plus usuelle pour les jeunes pianistes (mais aussi pour les pianistes avancés), que celle qui consiste à poser un $2^{\mathrm{e}}$ doigt à la main droite accompagné d'un $3^{e}$ à la main gauche. En effet, cette première configuration de doigtés se retrouve dans tous les manuels de 
piano (à commencer par les doigtés des gammes) et renvoie en partie aux principes de symétrie de l'arrangement des doigts que développe Fétis (1840) dans sa méthode. Lauteur met en rapport la symétrie dans l'arrangement des doigts avec la symétrie dans l'arrangement des notes, ce qui selon lui a «pour effet de faciliter l'exécution » (p. 29). Ce principe est aussi renforcé par l'idée qu'elle permettrait selon un professeur une meilleure mémorisation (cf. tableau 1, P6), mais aussi que ce choix dépend du saut qui précède.

Cette dernière assertion, qui indique que le choix du doigté dépendrait aussi de ce qui précède, mais aussi de ce qui suit, renvoie à la question de la délimitation de la tâche, des critères de cette segmentation, mais aussi au problème plus global de la caractérisation de la tâche. On pourrait en fait concevoir, en s'appuyant sur les différentes justifications apportées par les acteurs, trois types de contraintes susceptibles d'intervenir dans le choix du doigté pour l'exécution de cet extrait musical :

- La première contrainte renvoie à l'enchaînement des mesures 2 et 3 . Le déplacement rapide latéral du ré au fa\# (sixte descendante) constitue une première contrainte apparentée à celle du « ciblage ». Étant donné que la note ré est jouée avec le $1^{\text {er }}$ doigt, il se pourrait que l'arrivée sur le fa\# soit facilitée par un doigt plus directeur qu'un autre et/ou que le mouvement contraire entre les deux mains sur ce saut soit facilité par un doigté en particulier. Notons également que le déplacement imposé privilégie, comme doigt le plus proche pour l'exécution, le second doigt, qui se retrouve placé avant le troisième.

- La deuxième contrainte se situe au premier temps de la mesure 3, et concerne l'exécution du groupe fa\#-sol-la. Ce groupe doit être exécuté assez rapidement (présence de doubles croches) et avec précision, raisons pour lesquelles certains éditeurs privilégient les doigts les plus agiles (234 au lieu de 345). Le choix du doigté pourrait ici se comprendre, comme nous l'avons développé, en raison de facteurs de coordinations bi-manuelles, en témoigne par la suite le renversement quantitatif des doigtés proposés par les éditeurs sur ce type de cellule : ils proposeront à l'unanimité l'exécution du groupe do\#-ré-mi (même rythme et même intervalle que la cellule considérée; mes.9) avec le second doigt.
- La troisième contrainte, concerne l'exécution des mesures 3 et 4 , et renvoie à la notion de position que nous avons préalablement explicitée: il s'agit de faire un choix entre une position de ré

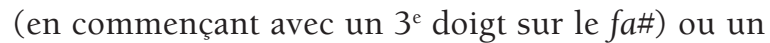
ajustement de la position par extension du pouce en fin de phrase (en commençant par un 2). Le fait de prendre en compte l'ensemble de la phrase, peut permettre l'émergence de tensions entre différents choix possibles : par exemple, si l'expert choisit de jouer le groupe fa\#-sol-la avec les doigtés 234 (les doigts les plus agiles), il se pourrait qu'il préfère finalement face à la phrase entière jouer les doigtés 345 (doigts moins agiles, mais qui permettent de jouer la phrase sur une position), la synchronisation des deux mains étant susceptible de favoriser également l'emploi de certains doigtés.

Ces situations ont été opérationnalisées dans des tâches expérimentales reprenant les variables précédemment citées (Bourg, 2006b), et la variable mode d'exécution à deux modalités (exécution main droite seule ou deux mains ensembles). Les tâches ont été ordonnées et présentées indépendamment les unes des autres à 6 pianistes. Les résultats indiquent que ce qui semble déterminer le doigté du fa\# ( $3^{\mathrm{e}}$ mesure), en l'occurrence le fait de mettre ou non un $3^{\text {e }}$ doigt, c'est l'exécution de la phrase complète, d'autant plus si elle est jouée en parallèle avec la main gauche. Le saut ne semble pas déterminer, sinon que faiblement, la sélection du doigté dans ce passage.

Cet exemple montre que le choix des doigtés diffère en fonction de l'institution dans laquelle on se trouve. Les différents acteurs apportent néanmoins les mêmes justifications par rapport à la pertinence de l'emploi de certains doigtés, même s'ils n'accordent pas finalement le même poids aux différentes composantes de la situation. Pour les experts, le choix du doigté renvoie le plus souvent à des aspects d'ordre esthétique. Dans cet extrait les experts privilégient ainsi les doigts agiles 234 dans le but d'obtenir un meilleur résultat sonore dans l'articulation des notes. Pour les éditeurs et professeurs de piano, le choix se tourne davantage vers des doigtés qui vont favoriser l'aisance technique de l'exécution pour les apprentipianistes. Ils choisissent ainsi plus systématiquement les doigtés 345 qui facilitent selon eux l'exécution de l'extrait : ils justifient leur choix majoritairement en s'appuyant sur la notion de position (tableau 1, P1, 
P2, P3, P6), mais aussi en invoquant les problèmes de coordination bi-manuelle (P5), de mémorisation (P6), et en référant à des aspects anatomiques et physiologiques (P4). Certains commentaires de professeurs montrent enfin que les doigtés qu'ils proposent ne sont pas forcément les leurs (P2, P6) et qu'ils essayent donc d'adapter ceux-ci en fonction de leurs élèves, parfois en invoquant d'ailleurs (P6) une recherche du doigté menée conjointement avec l'élève. Plus généralement, on pourra distinguer parmi les pratiques des éditeurs, ainsi que celle des professeurs, des intentions didactiques qui se situent : soit au niveau de la résolution de la tâche dans son ensemble (les doigtés proposés permettent à l'élève d'exécuter globalement le morceau); soit au niveau de la technique visée (les doigtés proposés permet- tent à l'élève d'apprendre de nouvelles techniques, techniques qui sont parfois accompagnées de leurs discours technologiques, tout en lui permettant de se construire une représentation des différents principes de cette pratique); dans ce deuxième cas le doigté ne constitue pas seulement un moyen (qui pourrait se confondre avec le but: jouer le morceau), mais devient un objet d'apprentissage. Par exemple, les éditeurs ou professeurs peuvent inciter les élèves à choisir leur doigté, en ne les inscrivant pas, ou du moins de manière non systématique (notamment pour des motifs réitérés, qui imposent alors à l'élève de mobiliser un schème lié à la reconnaissance d'une configuration mélodico-rythmique et de son doigté), ou encore peuvent proposer plusieurs doigtés pour une même tâche.

\begin{tabular}{|c|c|}
\hline Commentaires des professeurs de piano concernant l'emploi de certains doigtés & $\begin{array}{l}\text { Notions/dimensions } \\
\text { invoquées }\end{array}$ \\
\hline $\begin{array}{l}\text { P1 : « Le fait de commencer sur } 3 \text { aux deux mains permet d'englober le tout ». } \\
\text { P2: « Moi, je mets un } 2 \text { (à la main droite), mais avec les élèves je mets plutôt un 3, ça permet de rester } \\
\text { dans la même position ». } \\
\text { P3 : « Le } 3 \text { c'est plus facile pour les élèves; ils restent sur la même position pour l'ensemble de la } \\
\text { phrase ». }\end{array}$ & - Position \\
\hline $\begin{array}{l}\mathrm{P} 4: \text { " Je conseille dans ce passage un } 2^{\mathrm{e}} \text { doigt (à la main droite), parce qu'une touche noire avec le } \\
\text { pouce c'est pas terrible, et avec le } 3^{\mathrm{e}} \text { doigt on utilise alors des doigts faibles. Le } 2^{\mathrm{e}} \text { permet une bonne } \\
\text { articulation ». }\end{array}$ & $\begin{array}{l}\text { - Adaptation morphologie } \\
\text { des doigts / topographie du } \\
\text { clavier } \\
\text { - Do ig t s forts / } \\
\text { articulation }\end{array}$ \\
\hline $\begin{array}{l}\text { P5: « Le } 3^{e} \text { doigt permet une bonne assise des deux mains, c'est plus facile de jouer avec } 3 \text { (main } \\
\text { gauche) }-3 \text { (main droite)». }\end{array}$ & $\begin{array}{l}\text { C o or d i n a t i o n } \\
\text { bi-manuelle }\end{array}$ \\
\hline 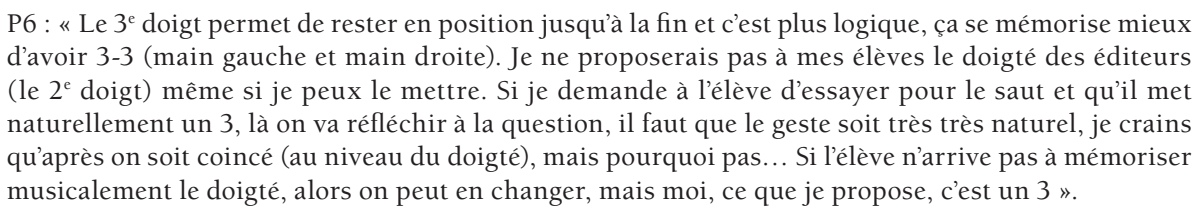 & $\begin{array}{l}\text { - Position } \\
\text { - Mémorisation } \\
\text { - Enchaînement inter } \\
\text { tâche }\end{array}$ \\
\hline
\end{tabular}

Tableau 1 : justifications apportées par les professeurs de piano sur l'emploi des doigtés

\section{Discussion}

Dans cette contribution nous avons notamment voulu interroger la pertinence en musique, de s'appuyer sur la notion d'organisation praxéologique, dans le cadre plus large de l'étude de phénomènes de transposition didactique. En effet, si la théorie de la transposition didactique a pu se heurter, à un moment donné, à quelques critiques qui se sont focalisées d'ailleurs, non sur les mécanismes de la transposition didactique, mais sur l'objet de ces mécanismes, le "savoir savant ", la notion de praxéologie semble pouvoir renouer avec certaines disciplines qui ne s'étaient pas reconnues dans cette première formulation théorique et pour lesquelles les «savoirs en acte » construits par l'expérience prédominent sur les connaissances explicitées dans des textes. On sera sensible concernant cette notion, au fait qu'elle permet de dépasser, comme le notent Bosch et Chevallard (1999), l'opposition structurelle 
qui existe dans la culture occidentale, entre les activités considérées comme «manuelles » et les activités dites « intellectuelles ». Les notions de tâche, technique, technologie et théorie, qui forment une organisation praxéologique, ont été proposées ainsi par Chevallard pour modéliser les pratiques sociales en général et l'activité mathématique en particulier. Dans le cadre de la pratique pianistique, nous avons présenté un ensemble d'institutions qui participe à la transposition des savoirs ou plus exactement des praxéologies. Lobjet considéré, le doigté, implique la mobilisation de techniques pianistiques particulières relativement à certains types de tâches, qui pourront se voir justifier de différentes façons en fonction des institutions. Nous aimerions aussi revenir sur la pertinence de l'utilisation des différentes composantes des praxéologies, pour la description et l'étude des conditions de réalisation de la pratique pianistique.

Nous avons observé à l'appui de quelques exemples qu'une tâche, amène à mettre en œuvre des techniques pianistiques différentes. Nous avons remarqué aussi, fidèlement aux remarques de Chevallard (1999b), en prenant pour exemple l'évolution du doigté dans les gammes, que ces techniques pouvaient varier dans le temps, de pays à pays, et plus généralement d'institution à institution. Ces techniques peuvent faire l'objet de discours - les technologies - qui ont pour fonction d'expliquer, d'éclairer, de justifier les techniques pianistiques. Nous avons cependant aussi rencontré certaines difficultés à éclairer les technologies par des théories. Si nous l'avons fait dans certains cas, c'est en nous centrant avant tout sur des aspects moteurs, et c'est en invoquant des savoirs scientifiques contributoires tels que par exemple certains principes d'ordre biomécanique. Il nous semble aussi que les tâches en musique ne peuvent être saisies complètement par une théorie, et que les théories auxquelles nous pourrions faire appel, ne participent pas de la même façon qu'en mathématiques à la construction d'une organisation praxéologique complète. Les théories dans la pratique pianistique sont invoquées, elles sont contributoires, elles n'engendrent pas spécifiquement des technologies et des techniques. Mais ce qui importe plus sûrement c'est de voir que certains discours endossent cette fonction, fonction technologico-théorique. Létude de ces types de discours, dans le cadre d'un élargissement des institutions étudiées, permettrait par exemple de reconsidérer la notion d' « école » dans la pratique instrumentale - on parle ainsi d' « école russe » de piano, d' « école française », etc. - notion que l'on a souvent défini d'un point de vue sociologique, institutionnel, mais sans véritablement entrer dans une analyse didactique des praxéologies. On pourrait analyser ainsi, pour un objet en particulier, les praxéologies qui seraient en fonction de ces écoles, dominantes, attendues, pertinentes, acceptables ou inacceptables.

Outre le niveau de la théorie, nous nous sommes heurtés à la question du niveau de précision de la caractérisation de la tâche. La distinction proposée par Chevallard (1999a) entre genre et type de tâche, qui distinguerait par exemple en mathématiques, calculer considéré comme genre de tâche, et calculer la valeur d'une fonction en un point comme type de tâche, ne nous semble pas assez précise pour le domaine que nous étudions, du moins tel que nous avons mené son étude. Si Chevallard pose qu'un type de tâche «suppose un objet relativement précis » (1999a, p. 224), le « relativement » laisse un certain flou qui pourrait constituer un trait faisant obstacle à la description des praxéologies. Par exemple, en posant comme genre de tâche « monter une gamme », nous pourrions considérer une multitude de types de tâches, pas seulement en indiquant la tonalité, comme « monter la gamme de do majeur», mais aussi en considérant la main qui joue, le rythme, le tempo, la note de départ et d'arrivée, la nature du passage, le cadre esthétique, le type de piano, l'acoustique de la salle, etc. Tous ces paramètres peuvent agir comme des variables potentielles, susceptibles de transformer la technique employée. Le tableau des gammes que nous avons présenté (cf. figure 4) ne précise pas d'ailleurs toutes les pratiques possibles formulées par les auteurs. Ainsi, Santa Maria (1565) nous énonce plusieurs cas de figures des doigtés (notamment pour les descentes) qui sont fonction du nombre de notes, du rythme, de la présence ou non d'une anacrouse. Considérant la dimension esthétique, soulignons qu'on ne jouera pas une gamme chromatique avec les mêmes doigtés et en mobilisant les mêmes techniques, en fonction que la tâche se trouve dans une partition de Beethoven ou dans une partition de Liszt. Considérant la dimension motrice, soulignons que « jouer une gamme dans un tempo rapide », qui correspond à un sous-ensemble de «jouer une gamme », fait intervenir des techniques différentes qui peuvent être en partie indépendantes 
des techniques de « jouer une gamme dans un tempo lent », car suscitant des réorganisations motrices importantes. Par exemple, sans toutefois s'attacher au doigté, Bernstein et de Popova (1930/2003) décrivent précisément les mouvements de pianistes, au cours de l'exécution d'une tâche, qui consiste à jouer en accélérant le tempo, mais aussi en changeant les dynamiques, des octaves parallèles répétées jouées avec une seule main. Dans leur expérience les auteurs interrogent la pertinence de l'expression « jouer avec du poids ${ }^{17}$. En terme biomécanique, ils cherchent à savoir si les mouvements sont dus à la force de la gravité ou à des forces additionnelles (musculaires). Les auteurs montrent d'abord que dans aucune des conditions expérimentales les forces au niveau de la main ne correspondent à un mouvement passif ( «jouer avec du poids» serait une expression qui tiendrait davantage de la métaphore), mais ils observent aussi, en fonction des variations de consigne (accelerando ou crescendo), des réorganisations des coordinations: dans un tempo lent, les pianistes jouent en faisant participer le mouvement de l'avantbras entier, dans un tempo rapide les pianistes jouent seulement sur la flexion du poignet (les mouvements de la main, par rapport aux mouvements de l'avantbras, seraient comparables à des oscillations élastiques forcées). Les auteurs montrent ainsi le rôle des contraintes biomécaniques qui en fonction des variations des paramètres de la tâche peuvent amener à des réorganisations motrices dues au potentiel d'auto-organisation du système moteur.

En lien avec le problème de caractérisation de la tâche, se pose celui de sa délimitation. Leétude de cas que nous avons soumis à l'analyse montre que toute tâche s'inscrit à l'intérieur d'autres tâches (tâches qui pourraient d'ailleurs certainement différer qualitativement), ce qui peut provoquer de multiples recadrages de la tâche. Ces recadrages varient en fait selon ce que les institutions veulent nous laisser voir, ce qui peut laisser souvent le contenu mal défini. Caractériser des tâches au piano, implique avant tout qu'on les appréhende de manière fortement contextualisée et dans toute leur complexité. Notre objet de savoir, le doigté, ne pourrait ainsi constituer une entité détachée de son contexte, qui se situe avant tout, dans le domaine de l'action et de l'interprétation : les énoncés propositionnels concernant le doigté ne se situent pas d'ailleurs dans le registre de la validité épistémique, mais plutôt dans le registre de la validité pragmatique (il existe de « bons » ou de « mauvais » doigtés), dans une perspective d'efficacité, de faisabilité, et de pertinence par rapport à des choix esthétiques. 


\section{NOTES}

1. On constatera l'absence de la didactique de la musique dans l'analyse épistémologique des didactiques disciplinaires que propose Sarremejane $(1998,2001)$, et sa récente mais timide réapparition dans le répertoire annexe des différentes didactiques adjoint au Dictionnaire des concepts fondamentaux des didactiques (Reuter, 2007).

2. Dichotomie proposée par G. Mialaret (1976).

3. Sur ce point, on remarquera que Martinand, dans la préface du Guide bibliographique des didactiques (Desvé, 1993) cité précédemment, constatant l'avancée des didactiques, énonce le programme possible d'une « didactique comparée », alors que le champ de la didactique de la musique n'est présenté encore que comme «potentiel ». Selon Martinand la « didactique comparée » pourrait comporter : « - des chronologies comparatives de disciplines, des périodisations parallèles, - des études comparées, des structures, fonctions, statut des disciplines dans les curriculums, - des analyses comparatives des 'objets de recherche', par exemple ces thèmes fréquents que sont l'erreur, le problème ou la tâche, les compétences, les représentations » (p. 15).

4. Nous rejoignons la position de Daunay (2007) et sommes attentifs à ses propos lorsque celui-ci se demande si toutes les didactiques sont prêtes pour le projet de didactique comparée, et indique qu' « il est à craindre que les didactiques émergentes, au lieu de prendre le temps de se constituer solidement sur leurs propres bases épistémologiques, se voient en partie contraintes par les autres didactiques et le projet comparatiste» (p. 77).

5. Nous retenons ici les ouvrages dans lesquels ont été développées les notions de ces deux auteurs. On retrouvera néanmoins leur apparition dans des écrits antérieurs : Chevallard (1978); Martinand (1981).

6. Par exemple, on se référera aux textes suivants émis par la direction de la musique, de la danse, du théâtre et des spectacles (DMDTS): Schéma directeur de l'organisation pédagogique des écoles de musique et de danse (1992); Schéma d'orientation pédagogique des écoles de musique et de danse (1996); Charte de l'enseignement artistique spécialisé en danse, musique et théâtre (2001); Schéma national d'orientation pédagogique de l'enseignement initial de la musique (2008).

7. Nous nous centrons sur l'étude d'un morceau en particulier afin de limiter le champ d'investigation. Ce morceau, la Musette en ré majeur (BWV Anhang 126), classiquement étudié dans les conservatoires, est extrait d'un recueil qui est connu sous le nom du Petit livre d'Anna Magdalena Bach (1725).

8. On nomme les doigts par un chiffre : pour les deux mains, on numérote les doigts de 1 à 5 , en partant du pouce jusqu'à l'auriculaire (pouce $=1$, index $=2$, majeur $=3$, annulaire $=4$, auriculaire $=5$ ).

9. Par exemple Debussy note dans la préface de ses Études, qu'« intentionnellement, les présentes Études ne contiennent aucun doigté ». Il en explique les raisons : «Imposer un doigté ne peut logiquement s'adapter aux différentes conformations de la main. La pianistique moderne a cru résoudre cette question en superposant plusieurs (sic.) ; ce n'est qu'un embarras... (...)»; puis conclut : « l'absence de doigté est un excellent exercice, supprime l'esprit de contradiction qui nous pousse à préférer ne pas mettre le doigté de l'auteur, et, vérifie ces paroles éternelles: 'On n'est jamais mieux servi que par soi-même'. Cherchons nos doigtés! » (Préface des Études, 1916).

10. On soulignera qu'il existe plusieurs types d'éditions, certaines se voulant être au plus près de la version originale de l'œuvre (les Urtext), d'autres s'octroyant la liberté de compléter la partition en ajoutant des doigtés, mais aussi notamment des signes d'articulations ou des nuances.

11. Le modèle de Parncutt et al. (1997) a cependant un domaine d'efficacité assez restreint : il permet en fait de prédire des combinaisons de doigtés uniquement sur de courts fragments musicaux écrits en langage tonal, joués legato, et pour une seule main. Certaines techniques sont également évacuées des prédictions du modèle : a) Les doigtés de substitution (lorsqu'on change de doigt sur une même note sans la rejouer); b) Le jeu legato avec un même doigt (on peut par exemple faire glisser un même doigt d'une touche noire à une touche 
blanche, ou encore faire « ramper » le pouce d'une touche blanche à une autre); c) Le jeu par chevauchement des doigts 3, 4 et 5 (notamment lorsqu'il s'agit d'exécuter des lignes mélodiques chromatiques, à l'image de l'Étude op. $10 n^{\circ} 2$ de Chopin).

12. Nous insistons ici sur le fait que nous nous concentrons sur la musique qui est transmise par l'écriture et qui se réalise au travers du médiateur principal que constitue la partition (il existe bien sûr des formes d'écritures extra-occidentales).

13. On notera qu'en France, l'enseignement musical est dispensé dans deux types de structures différentes : il existe d'une part les établissements scolaires où on enseigne l' «éducation musicale », et d'autre part les établissements spécialisés qui enseignent la pratique instrumentale. Dans ce dernier cas, la pratique instrumentale peut être enseignée dans différentes institutions qui ont des statuts et des exigences particuliers : il existe notamment les CNSM (Conservatoire National Supérieur de Musique de Paris et de Lyon) qui s'adressent aux futurs professionnels de la musique, les CRR et CRD (Conservatoires à Rayonnement Régional ou Départemental; anciennement CNR et ENM), moins prestigieux que les CNSM et plus nombreux, restent cependant à visée professionnelle, et les CRC et CRI (Conservatoires à Rayonnement Communal ou Intercommunal; anciennement EMMA et CMMA) qui s'adressent au grand public et dont la visée de la pratique instrumentale est plus ludique (voir Ganvert, 1999, pour une description détaillée de l'enseignement musical en France).

14. Un peu plus tard, cet usage du jeu inégal nous est rapporté par Couperin (1717), qui distingue le jeu français, du jeu italien : "Il y à selon moy dans notre facon d'écrire la musique, des déffauts qui se raportent a la manière d'écrire notre langue! C'est que nous écrivons diffèremment de ce que nous exécutons : ce qui fait que les étrangers jouent notre musique moins bien que nous ne fesons la leur. Au contraire les Italiens écrivent leur musique dans les vrayes valeurs qu'ils l'ont pensée. Par exemple nous pointons plusieurs croches de suites par degrés conjoints; Et cependant nous les marquons égales! notre usage nous a asservis; et nous continuons.» (p. 39-40).

15. Nous qualifions d'expert des pianistes qui ont obtenu un $1^{\text {er }}$ prix de piano (dans un CRR ou dans un CNSM) et qui se produisent régulièrement en public dans un répertoire soliste.

16. Les professeurs de piano interrogés ont obtenu, suite à un premier prix, un des diplômes spécialisés d'enseignement (le Diplôme d'État ou le Certificat d'Aptitude). Certains ont suivi une formation initiale dans un Centre de formation à l'enseignement de la danse et de la musique (CeFEDeM) et tous enseignent dans des conservatoires municipaux depuis au moins cinq ans.

17. Il s'agit d'une expression qui est couramment employée dans l'enseignement du piano, qui renvoie au fait que l'exécutant doit jouer "dans le clavier », et non de manière superficielle. Fassina (2000) distingue à juste titre « le poids du bras qui ne varie pas » et « la force du bras à laquelle correspond une énergie qui varie, elle, et que l'on dose» (p. 44).

\section{BIBLIOGRAPHIE}

Adam, L., \& Lachnitz, L.-W. (1798/1801). Méthode ou principe général du doigté pour le forte piano. Suivie d’une collection complette (sic.) de tous les traits possibles avec le doigté en commençant par les plus aisés jusqu'aux plus difficiles; terminé par un dictionnaire de passages aussi doigtés tirés des auteurs les plus célèbres ( $1^{r e}$ et $2^{e}$ partie). Paris : Sieber.

Bach, C.P.E. (1753/1979). Essai sur la vraie manière de jouer des instruments à clavier. (D. Collins, Trad.). Paris : J.-C. Lattès. (Édition originale, 1753).

Badura-skoda, P. (1974). L'art de jouer Mozart au piano. Paris : Buchet/Chastel.

Bermudo, J. (1555/1957). Declaracion de instrumentos musicales. Osuna (Facs. 1957, Kassel : Bärenreiter-Verlag). 
Bernstein, N.A., \& Popova, N.S. (1930/2003). Studies on the biomechanichs of the piano strike. Motor control, $7(1), 3-45$.

Bosch, M., \& Chevallard, Y. (1999). Ostensifs et sensibilité aux ostensifs dans l'activité mathématique. Recherche en didactique des mathématiques, 19(1), 77-124.

Boudinet, G., \& Fijalkow, C. (Eds.). (2005). Mélanges pour Jean-Pierre Mialaret - De la fondation des Sciences de l'éducation musicale... Paris : L'Harmattan.

Bourg, A. (2006a). Analyse comparative des notions de transposition didactique et de pratiques sociales de référence : Le choix d'un modèle en didactique de la musique? Journal de Recherche en Éducation Musicale, 5(1), 79-116.

Bourg, A. (2006b). Phénomènes de transposition autour de deux cuvres extraites du Petit livre d'Anna Magdalena Bach: Des pratiques pianistiques du doigté à leur enseignement. Thèse de doctorat, Université Paris Descartes, Paris.

Bourg, A. (2007). Didactique du piano : Phénomènes de transposition autour d'une ouvre extraite du Petit livre d'Anna Magdalena Bach. In D. Pistone (Ed.), Pianos \& pianistes dans la France d'aujourd'hui (pp.101-112). Presses Universitaires de la Sorbonne, série Conférences et Séminaires, 29.

Breithaupt, R.M. (1921). Die natuerliche klaviertechnik. Leipzig : C.F. Kahn.

Büchner, J. (1520/1974). Fundamentum. Zürich. (Facs. 1974, Frankfurt : Henry Littolff).

Chevallard, Y. (1978). Sur la transposition didactique dans l'enseignement de la statistique. In Didactique de la statistique (chap. 6). Notes internes, IREM d'Aix-Marseille et CNAM (Paris).

Chevallard, Y. (1985/1991). La transposition didactique : du savoir savant au savoir enseigné. Grenoble : La pensée sauvage.

Chevallard, Y. (1996). La fonction professorale : esquisse d'un modèle didactique. In R. Noirfalise \& M.J. Perrin Glorian (Eds.), Actes de la VIII école d'été de didactique des mathématiques 22-31 aout 1995 (pp.82-122). Clermond-Ferrand : IREM de Clermond-Ferrand.

Chevallard, Y. (1999a). Lanalyse des pratiques enseignantes en théorie anthropologique du didactique. Recherches en Didactique des Mathématiques, 19(2), 221-266.

Chevallard, Y. (1999b). La recherche en didactique et la formation des professeurs : problématiques, concepts, problèmes. Actes de la Xe école d'été de didactique des mathématiques (Houlgate, 18 au 25 août 1999), 98-112.

Chevallard, Y. (2007). Éducation et didactique : une tension essentielle. Éducation \& didactique, 1(1), 9-27.

Clementi, M. (vers 1801). Méthode pour le piano Forte - Contenant, les éléments de la musique, et des leçons préliminaires, sur le doigté, accompagnées d'exemples, et suivies de cinquante leçons doigtées, par les compositeurs les plus célèbres. Paris : Pleyel.

Corette, M. (1749). Les amusements du parnasse méthode courte et facile pour apprendre à toucher le clavecin. Avec les plus jolis airs à la mode où les doigts sont chiffrés pour les commençans. Ensemble des principes de musique. Livre Ier. Paris : l'Auteur, Boivin, Le Clerc.

Couperin, F. (1717). L'art de toucher le clavecin. Paris : l'Auteur, Foucault.

Daunay, B. (2007). Didactique comparée. In Y. Reuter (Ed.) Dictionnaire des concepts fondamentaux des didactiques (pp.75-78). Bruxelles : De Boeck Université.

Desvé, C. (Ed.) (1993). Guide bibliographique des didactiques : Des ressources pour les enseignants et les formateurs. Paris : INRP.

Diruta, G. (1593/1984) Seconda parte del Transilvano dialogo. Venise : Giacomo Vicenti (Facs. 1984, Henryville : Institute of Mediaeval Music).

Duphly, J. (1769). Du doigter : manuscrit autographe figurant sur la page de garde d'un exemplaire du premier livre de clavecin (Pièces de clavecin dédiées à monseigneur le Duc d'Ayen, Paris : Vandome) appartenant à Lord Fitzwilliam.

Fassina, J. (2000). Lettre à un jeune pianiste. Paris : Fayard.

Fétis, F. (1840). Méthode des méthodes de piano: Traité de l'art de jouer de cet instrument basé sur l'analyse des meilleurs ouvrages qui ont été faits à ce sujet et particulièrement des méthodes. Paris : Schlesinger. 
Francès, R. (1974). L'enseignement programmé de la musique. Principes d'une structuration de la matière et résultats d'un programme de solfège élémentaire. Sciences de l'Art, IX(1-2), 121-137.

Ganvert, G. (1999). Lenseignement de la musique en France. Paris : L'Harmattan.

Hondré, E. (Ed.) (1995). Le conservatoire de musique de Paris : regards sur une institution et son histoire. Paris : Association du bureau des étudiants du Conservatoire national supérieur de musique.

Hummel, J. N. (1828/1982). Méthode complète théorique et pratique pour le piano-forte. Wien : Haslinger. (Facs. 1982, Genève : Minkoff).

Johsua, S. (1998). Des « savoirs» et de leur étude : vers un cadre de réflexion pour l'approche didactique. L'Année de la recherche en sciences de l'éducation, 79-97.

Kaemper, G. (1968). Techniques pianistiques - Lévolution de la technologie pianistique. Paris : Leduc.

MacKenzie, C.L., \& Van Eerd, D.L. (1990). Rhythmic precision in the performance of piano scales : motor psychophysics and motor programming. In M. Jeannerod (Eds.), Attention and performance 13 : motor representation and control (pp.375-408). Hisdale : Lawrence Erlbaum Associates.

Marpurg, F.W. (1750). LArt de toucher le clavecin selon la manière perfectionnée des modernes. Paris : Naderman, Le Menu.

Martinand, J.-L. (1981). Pratiques sociales de référence et compétences techniques : propos d'un objet d'initiation aux techniques de fabrication mécanique en classe de quatrième. In A. Giordan (Ed.), Diffusion et appropriation du savoir scientifique. Actes des troisièmes journées internationales sur l'éducation scientifique (pp.149-154). Paris : Université Paris 7.

Martinand, J.-L. (1986). Connaître et transformer la matière. Paris-Berne : Peter Lang.

Marsenach, J., \& Amade-Escot, C. (1993). Les orientations de la recherche en didactique de l'éducation physique et sportive. Revue Française de Pédagogie, 103, 33-42.

Mialaret, G. (1976). Les Sciences de l'Éducation. Paris : PUF.

Mialaret, J.-P. (1979). Apprentissage musical et enseignement programmé. Monographies françaises de psychologie, 47. Paris : CNRS.

Nonot, J. (1797). Leçons méthodiques de clavecin ou de forte-piano. Paris : Richomme.

Ortmann, O. (1929). The physiological mechanics of piano technique. Londres et New York : E.P. Dutton.

Parncutt, R., Sloboda, J.A., Clarke, E., Raekallio, M., \& Desain, P. (1997). An ergonomic model of keyboard fingering for melodic fragments. Music Perception, 14(4), 341-382.

Parcnutt, R., Sloboda, J.A., \& Clarke, E.F. (1999). Interdependence of right and left hands in sight-reads, written, and rehearsed fingerings of parallel melodic piano music. Australian Journal of Psychology, 51(3), 204-210.

Pleyel, I., \& Dussek, J.-L. (1797). Méthode pour le piano forte contenant les principes du doigté de clavecin. On y trouvera aussi une nouvelle manière d'accorder le forte piano. Paris : Pleyel.

Raisky, C., \& Caillot, M. (Eds.). (1996). Au-delà des didactiques, le didactique : Débats autour de concepts fédérateurs. Paris-Bruxelles : De Boeck et Larcier S.A.

Reuter, Y. (2007). Dictionnaire des concepts fondamentaux des didactiques. Bruxelles : De Boeck Université.

Robine, M. (2006). Analyse de la performance musicale et synthèse sonore rapide. Thèse de doctorat, Université Bordeaux I, Bordeaux.

Rogalski, J., \& Samurçay, R. (1994). Modélisation d'un « savoir de référence » et transposition didactique dans la formation de professionnels de haut niveau. In G. Arsac, Y. Chevallard, J.-L. Martinand, \& A. Tiberghien (Eds.), La transposition didactique à l'épreuve: Un analyseur pour la transposition didactique entre pratiques de référence et activités scolaires (pp.35-72). Grenoble : La pensée sauvage.

Saint-Lambert, M. de (1702). Les prinicpes du clavecin contenant une explication exacte de tout ce qui concerne la tablature et le clavier, suivi de Nouveau traité de l'accompagnement du clavecin, de l'orgue et des autres instruments. Paris : C. Ballard.

Santa Maria, T. de (1565/1972). Libro llamado Arte de tañer fantasía. Valladolid (Facs. 1972, Bergstrasse : Gregg international publishers).

Sarremajane, P. (1998). Contribution à l'histoire sémantique des didactiques disciplinaires, 1960-1995. Thèse de doctorat, Université Paris Descartes, Paris. 


\section{Adrien Bourg}

Sarremejane, P. (2001). Histoire des didactiques disciplinaires. Paris : L'Harmattan.

Schillinger, A. (1995). De la musique à l'éducation musicale. In M. Develay (Ed.), Savoirs scolaires et didactiques des disciplines (pp. 203-255). Paris : ESF.

Tetzel, E. (1927). Der anschlag beim klavierspiel in mechanisher und physiologischer hinsicht. Zeit für musikwissenschaft, octobre.

Venegas de Henestrosa, L. (1557/1944). Libro de cifra nueva. Alcalà : J. de Brocar. (Facs. 1944, Barcelone : H. Anglès).

Verret, M. (1975). Le temps des études (Thèse de 3e cycle, Université Paris Descartes, Paris, soutenue le 29 mai 1974). Paris : Librairie Honoré-Champion.

Viguerie, B. (1796). L'art de toucher le piano-forte. Paris : l'Auteur.

Wagner, C. (1988). The pianist's hand : anthropometry and biomechanics. Ergonomics, 31(1), 97-131. 